\title{
A Pilot Study Verifying How the Curve Information Impacts on the Driver Performance with Cognition Model
}

\author{
Xiaohua Zhao, Wei Guan, and Xiaoming Liu \\ Key Laboratory of Traffic Engineering, Beijing University of Technology, Beijing 100124, China \\ Correspondence should be addressed to Xiaohua Zhao; zhaoxiaohua@bjut.edu.cn
}

Received 18 September 2012; Revised 27 December 2012; Accepted 28 December 2012

Academic Editor: Wuhong Wang

Copyright ( 2013 Xiaohua Zhao et al. This is an open access article distributed under the Creative Commons Attribution License, which permits unrestricted use, distribution, and reproduction in any medium, provided the original work is properly cited.

\begin{abstract}
Drivers' misjudgment is a significant issue for the curve safety. It is considered as a more influential factor than other traffic environmental conditions for inducing risk. The research suggested that the cognition theory could explain the process of drivers' behavior at curves. In this simulator experiment, a principle cognition model was built to examine the rationality of this explanation. The core of this pilot study was using one of the driving decision strategies for braking at curves to verify the accuracy of the cognition model fundamentally. Therefore, the experiment designed three treatments of information providing modes. The result of the experiment presented that the warning information about curves in advance can move the position of first braking away from curves. This phenomenon is consistent with the model's inference. Thus, the conclusion of this study indicates that the process of the drivers' behavior at curves can be explained by the cognition theory and represented by cognition model. In addition, the model's characteristics and working parameters can be acquired by doing other research. Then based on the model it can afford the advice for giving the appropriate warning information that may avoid the driver's mistake.
\end{abstract}

\section{Introduction}

The safety at curves is an important issue for drivers as well as traffic engineers. Many investigations and researches had reported that a huge number of crashes or accidents happen at curves every year $[1,2]$. The causation of this phenomenon is that while the vehicle passing through a curve, the additional centripetal force will exert on the car. That force will induce a difficult driving task required the driver to complete [3], then drivers may easily make a mistake and cause an accident. To handle this problem, many traffic facilities, in-vehicle systems, and standards had been invented (or specified) to improve driving environment and curve safety $[4,5]$. Although these measures made curve driving safer, there are still a lot of crashes or accidents caused by drivers. Thus, many researchers have shifted their focus on studying the process of drivers' behavior at curves [6]. Moreover, based on the results/findings of those studies, engineers may improve or modify the measures to promote the curve safety. The characteristics of curve safety and driving models are two main basic elements of the researches.

Curve safety research takes a large portion of traffic studies. According to researches, a higher vehicle speed may largely increase the probability of crashes, but the direct reason which lead to accidents is always the drivers' incorrect operation $[7,8]$. While a vehicle is passing through curves, it requires more attention resources to collect information, more mental sources to make decisions, and more operations to perform as quickly and preciously as possible for drivers. High vehicle speed decreased the required time of the whole working process for drivers, which means drivers have to finish the process more quickly. As a result, the error tolerance decreased, and the accident possibility was increased. Comte and Jamso's study also showed that a large proportion of curve accidents were caused by a driver traveling too fast through a curve and then losing control of the vehicle or being forced into a skid and cannot perform the right operation 
[9]. In addition, it was a consensus that increasing degrees of curvature caused more accidents [10]. Another study found that the driver's correct expectation to an encountered curve could make driving safer [11].

In all, the key point to improve curve safety was to let the drivers know the information of coming curves well, then they can be attentive to perceive traffic information accurately, make appropriate decisions easily, and be prepared for more driving operations. Specifying the driver's working process clearly was the precondition for providing the appropriate information to drivers.

According to the cognition theory, driving performance is consisted by 3 phases: information perception, driving decision, and operation execution [12]. In addition, both phases of driving decision and operation execution partly rely on the information coming from the phase of information perception. Therefore, the information perception is the basis for the cognition. In this phase, the driver transforms the picture of real world into information that will be used in the other two phases. The more exact the transformed information was, the easier the driver can make appropriate decisions and correct operations.

Although the driver has five key perception systems, the visual system takes a considerable percentage in driving task [13]. Based on the visual system the driver would rebuild a driving scenario in their brain. The scenarios may reflect the past, current, and future driving situations. According to all the three scenarios drivers can decide their driving strategy. Thus, acquiring exact and valid information is the most important guarantee for curve driving safety. However, factors like environment, driver ability, or distraction may cause the acquisition of inexact and invalid information. As studies shown, most collisions were due to drivers' misperception of frontal curves [14] or failing to obey traffic signs [15]. Also, there is a consentaneous finding: while a driver is in the attention status or has received a warning [16], the probability of an accident is prominently reduced. In addition, Roca Javier et al.s. research states that if one wants to finish a driving task well, he must properly perceive traffic objects (e.g., road signs), maintain an appropriate state of alertness to make decisions, and perform the operation at the right time [17].

In summary, the objective of this paper is using the cognition theory to build a principium model to describe curve driving. This model is driven by information, and different information should induce different operation. To verify the accuracy of the cognition model and related assumption, we compared the driving operations with three different information origins. The result indicates that the curve driving behavior can be well explained by cognition model.

\section{Cognition Model}

Building a cognition model for the curve driving was the primary task in this study. However, the goal of this model was neither to simulate the driver's behavior accurately nor to rebuild the whole working process of the human brain. This study mainly focuses on representing an equivalent model for curve driving.

2.1. Cognition Theory. Cognition theory derived into two basic subjects: psychology and neurology. The former one focuses on studying the subjective human (just like how human think, why they have self-awareness), and provide a lot of basic theoretical and rich practical materials for cognition; the latter one is the study of objective human existence; through experiments it verifies a large number of assumptions for cognitive principles. Therefore, the cognition study may involve in both the subjective and objective aspects of human, which can give a comprehensive principle frame for explaining human behaviors.

At present, cognition theory has already been applied in several fields of study, and in the case of different studying priorities, the researchers had given the definition respectively from different academic areas. The followings are three representative definitions:

(1) cognitive architectures are designed to simulate human intelligence in a human-like way [18];

(2) a scientific hypothesis about those aspects of human cognition those are relatively constant over time and relatively independent of task [19];

(3) cognition is a term referring to the mental processes involved in gaining knowledge and comprehension, including thinking, judging, remembering, and problem solving [20].

Based on these definitions, this paper argues that the core of cognition theory is "studying how human respond to the objective/subjective things and the correlative characters, rules and principles". In addition, the final goal of cognitive studying is to build an equivalent model to represent how human respond to the objective/subjective things. Furthermore, the two contents of cognition model are cognition architecture and cognition process, as the following specification.

Cognition architecture can be defined as a set of functional modules, which are integrated in a specific order. The research about cognition architecture includes the modules' characters and their relationship. Generally, the modules include attention, memory, problem solving, decisionmaking, learning, and so on.

Cognition process refers to a temporal logical sequence to accomplish a cognitive task, which is based on specific cognition architecture. Some people claim that knowledge is the foundation of the cognition process [21], while others believe that the cognitive process is driven by events [22]. This paper assumes that information is the fundamental element in cognition process. Because every subjective and objective thing had uniform and inherent property, that is, information. In other words, everything is either a subjective or an objective representation of information. For example, the real curve warning sign on the roadway can be seen as objective representation of information of "there is a curve ahead"; yet when a driver knows the meaning of the traffic 
sign it converts to subjective reproduction in the driver's mind.

For this assumption, we can unify all kinds of things in the cognition model under a uniform measurement and consider various factors effectively. Before analyzing the factors affecting driving behavior, it can convert traffic sign, road condition, driving knowledge, or other things all into the information, which may help studying.

For this assumption, we can unify all kinds of things in the cognition model under a uniform measurement and consider various factors effectively. Before analyzing the factors affecting driving behavior, it can convert traffic sign, road condition, driving knowledge, or other things all into the information.

2.2. Model for Curve Driving. According to cognition theory, the cognition architecture of this model is composed by five kinds of general modules, involving perception module, transmission module, memory module, process module, and motion module. Each kind of modules has its own characters and function in different cognition models. The modules in this study can be described as follows.

(i) Perception module: obtain traffic information from the external environment. There are five kinds of perceptions for human: visual, aural, tactual, olfactory, and gustatory. This study only makes use of visual perception for testing cognition model.

(ii) Process module: convert the input information to output information. The conversion can described as productions or functions. The core process in this study is driving decision module. It can convert traffic information to the operation command information basing on the driving strategy. The driving strategy will be specified in Section 2.3.

(iii) Memory module: this module can be classified into two categories, short-term memory and long-term memory. The short-term memory was employed to store information which the process module's need, and the long-term memory was employed to store different kind knowledge library. This study involves both two kinds of memory modules. Short-term memory includes image buffer, knowledge buffer and constrain buffer, and long-term memory is the knowledge library, seen Figure 1.

(iv) Motion module: perform the command information coming from driving decision module.

(v) Transmission module: transmit information between different modules.

Based on modules mentioned above the cognition architecture can be represented as Figure 1.

Figure 1 has four kinds of information channels, and the channels are tagged by different arrows and box styles as the legend shown. The white boxes are the start or the end of the channels and each channel has its own function as described below. (i) The perception channel is used to collect information from traffic environment. The information of traffic environment is regarded as an image. Through the visual module and transmit module, it is stored in the image buffer as other analog signals. The visual encoder module converts those analog signals to abstract traffic information through the transmit module, and it will be used in the decision module. (The working process of other perception channels functions in the same way, this study will not focus on them.)

(ii) The motion channel is used to generate driving operation. When a driver makes a decision, it sends the command information to motor modules to perform through this channel.

(iii) The learning channel through the learning system transmits information from message buffers into the knowledge library.

(iv) The memory channel is used to convert the information from knowledge library to short-term memories. In this study, the goal analysis module selects the related knowledge according to the main driving goal, then puts them into constraint buffer and knowledge buffer for the process modules.

Although this architecture is simpler than the real driver's working process, it is still too complex for this pilot experiment to test all parts. It needs a further predigest by giving some constraint. The simplified model is shown in Figure 2.

In this model, the driving decision module has two information sources: one is visual perception; the other is knowledge library. The driving decision module makes a decision in accordance with information from these two sources. Then the motor system should output operation according to the decision. The different information input from two sources can eventually lead to different driving operations. Compared with Figure 1, the module in Figure 2 reduces the other perception channels, encoder module, constraint buffer, and transmit modules. Provided that all modules are maintained at a constant level, the output change is only caused by the two information sources. Therefore, we can simplify Figure 1 to Figure 2.

Based on the architecture, the cognition process is shown in Figure 3. The process starts from the image of traffic environment (seen as input) and ends with driving behavior (seen as output). Through the three phases: information acquiring, decision making, and driving operation, the three processes are executed parallel while the information is serial. It means at a particular moment, the decision-making process is caused by the previous visual perception.

2.3. Model Analysis. Based on the cognition theory, this study argued three assumptions about the cognition model and designed three information providing treatments. Under these conditions, the study makes the inference about the model to verify the rationality with the simulator experiment.

Assumption One: the strategy of driving decision module is that once the information "there is a curve ahead" is 


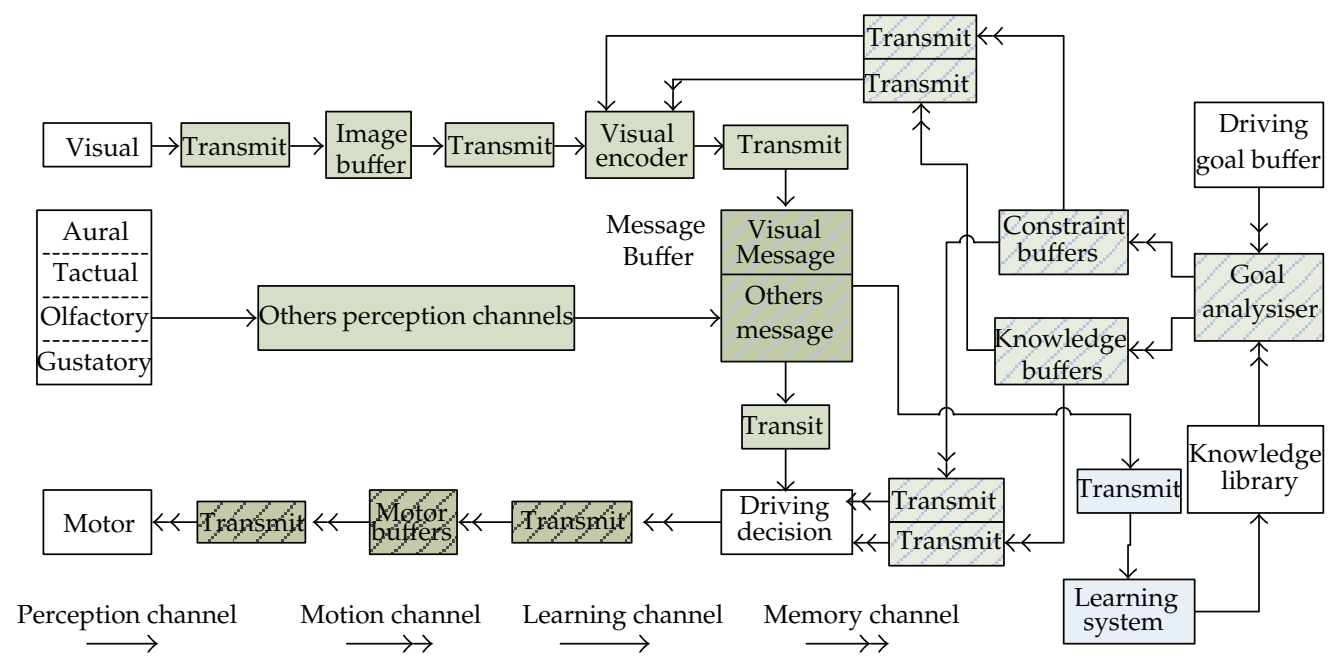

FIgURE 1: The architecture of cognition model.



FIGURE 2: The simplified cognition model.



Figure 3: The cognition process of the model.

confirmed and vehicle speed is higher than the limitation of passing curve, then the braking operation will be taken.

Assumption Two: the acquired information has a confidence value. If the sum of the confidence values with the same information is over a limen, this information can be confirmed as true.

Assumption Three: information acquired from visual perception channel can be partly stored in the knowledge library via learning system. The stored content may be enhanced by repeating.
Treatment A: the curve information without advanced warning information provided via visual perception channel.

Treatment B: the curve information with advanced warning information provided via visual perception channel.

Treatment $C$ : the curve information provided via visual perception channel, and the advanced warning information provided via memory channel from the knowledge library.

Inference: in the treatment $\mathrm{A}$, the decision module takes more time than other two treatments to confirm the curve information, then the model will brake at last. Since the decision module lacks the warning information, it has the lowest confidential value about the curve information. Furthermore, the higher confidence value may appear to confirm the information and do earlier braking operation.

\section{Methods}

3.1. Participants. All drivers are at the same level for this study. 18 male participants, having had their license for at least 2 years (average $=4.13, \mathrm{SD}=1.2$ ), aged 22-32 (average $=27.8, \mathrm{SD}=2.24)$, were recruited from the public. None of the subjects had color vision deficiencies. All of them possess normal or corrected-to-normal vision.

3.2. Apparatus. It is an appropriate choice to use the simulation experiment in this study mainly for three reasons: (1) keeping all trials under the same conditions; (2) making the experiment repeatable; (3) protecting participants from risk. 


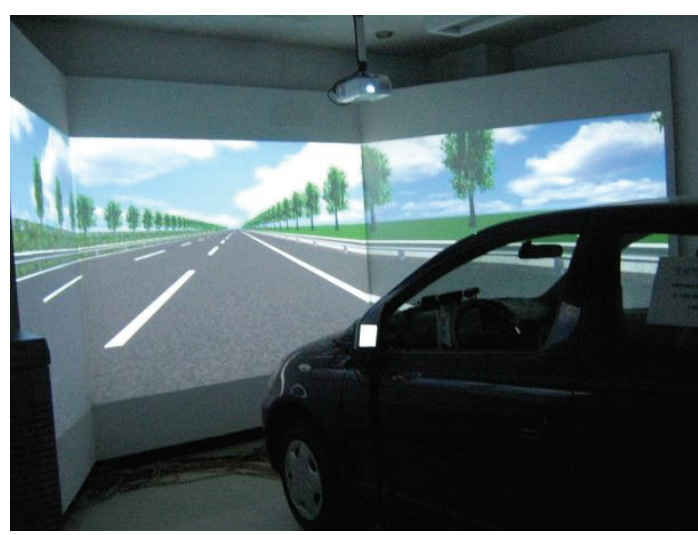

Figure 4: The AutoSim simulator system.

The whole experiment made use of the AutoSim simulator system (see Figure 4) at the laboratory of the Transportation Research Center at Beijing University of Technology.

The hardware is consisted by a vehicle, eight-networked computers (one is the master computer, one communicates with the vehicle system, and the other six are used to compute six different real-time views), a motion control device, and other equipment (such as video and audio devices). Beside, three main softwares used in the simulator experiment: Evariste (used to create experimental scenarios), Simword (for controlling the scenarios), and Scancer (for collecting data and generating car motion). The simulator can record data during the experiment including the action of the accelerator/brake pedal, the steering wheel, vehicle speed, and so forth. The frequency of recording is $30 \mathrm{~Hz}$.

3.3. Experimental Design and Procedures. In order to test the rational of cognition model, the simulator experiment must keep the same constraints with the model declared. The simulator environment can avoid interference or other uncertainties in the actual environment.

First, considering that there is more than one strategy for drivers making choices in driving, the most important constraint to be controlled is to make sure that drivers take the driving strategy, as Assumption one mentioned, when they encounter with the curve. If some other factors did not fit the condition of this strategy, the drivers may not follow that strategy. To access to this goal, the scenarios of this simulator experiment are designed as a single lane of rural roadway with no traffic flow. Considering that the vehicle needs to reach a higher speed than the limitation before entering into the curve, we designed an $800 \mathrm{~m}$ straight lane ahead of each curve for drivers' accelerating.

Second, the drivers' ability and driving state should also be under control. According to Figure 1, there are many kinds of factors affecting the driving operation. So when recruiting the participants, all the following characters are considered (age, gender, education, and driving frequency) to avoid these kinds of discomfort. Additionally the operator will give participants an instruction to ensure the driving task for experiment before driving.

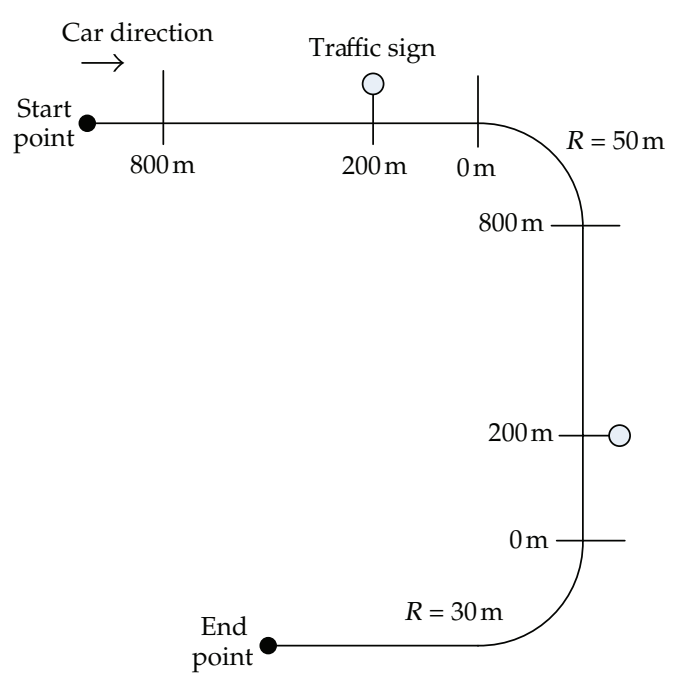

FIGURE 5: Five position of the traffic sign before the curves.

Third, corresponding to three the information providing treatments for the cognition model, 18 participants were divided into three groups randomly in this experiment. In group A, participants drove through curves without traffic sign providing warning information. In group $B$, participants drove through curves with traffic sign providing warning information. In group $\mathrm{C}$ participants drove through curves without traffic sign, but were provided with warning information via the map of scenario before driving. We designed two scenarios (shown in Figure 5), and the difference between them is whether there is a traffic sign before curves. Each scenario has two curves of which the radii are $30 \mathrm{~m}$ and $50 \mathrm{~m}$, with a length of $47.1 \mathrm{~m}$ and $78.5 \mathrm{~m}$, respectively. The position of traffic sign is $200 \mathrm{~m}$ away from the curve.

Finally the procedure of this experiment is stated as follow.

(i) Filling in basic information to acquire the characters information and driving state about drivers.

(ii) Participants have had a driving test to avoid drivers' making mistake because they are unfamiliar with the simulator.

(iii) When the drivers finished the driving test, the operator read the guidance to the driver and gave him the map (in group C).

(iv) After that, the drivers started the formal experiment, and each participant should drive repeatedly for five times, and there would be a rest for 2-3 minutes between the laps.

\section{Result}

According to the cognition model, the position of braking start is related with the time when the information "there is a curve ahead" is confirmed. The data of two kinds of braking operations (the first position to release the accelerator pedal, FPRA; the first position to press the brake pedal, FPPB) are used to verify the inference mentioned above. 
TABLE 1: The result of braking data of two curves.

\begin{tabular}{|c|c|c|c|c|c|c|c|c|}
\hline \multirow[b]{2}{*}{ Lap 1} & \multicolumn{2}{|c|}{ FPRA-R30 } & \multicolumn{2}{|c|}{ FPPB-R30 } & \multicolumn{2}{|c|}{ FPRA-R50 } & \multicolumn{2}{|c|}{ FPPB-R50 } \\
\hline & Average & SD & Average & $\mathrm{SD}$ & Average & SD & Average & SD \\
\hline Group-A & 104.78 & 32.19 & 86.51 & 34.64 & 103.89 & 51.31 & 72.40 & 34.26 \\
\hline Group-C & 192.10 & 108.60 & 117.18 & 66.95 & 184.97 & 57.61 & 145.36 & 58.49 \\
\hline Group-B & 197.44 & 28.25 & 158.94 & 42.51 & 185.28 & 60.17 & 169.11 & 87.31 \\
\hline Lap 2-5 & Average & SD & Average & SD & Average & SD & Average & SD \\
\hline Group-A & 160.07 & 58.76 & 123.49 & 37.17 & 188.41 & 67.10 & 151.09 & 69.78 \\
\hline Group-C & 201.22 & 66.27 & 164.16 & 46.36 & 199.28 & 47.23 & 166.32 & 45.24 \\
\hline Group-B & 218.80 & 49.86 & 188.48 & 39.44 & 253.48 & 72.88 & 216.59 & 69.73 \\
\hline
\end{tabular}

TABLE 2: The confidence value of different conditions.

\begin{tabular}{|c|c|c|c|c|}
\hline & Curve & $\begin{array}{l}\text { Traffic } \\
\text { sign }\end{array}$ & Memory & Total \\
\hline $\begin{array}{l}\text { Group A, } \\
\operatorname{lap} 1\end{array}$ & $\mathrm{~A} 1$ & 0 & 0 & $\mathrm{~A} 1$ \\
\hline $\begin{array}{l}\text { Group B, } \\
\text { lap } 1\end{array}$ & $\mathrm{~A} 1$ & B1 & 0 & $\mathrm{~A} 1+\mathrm{B} 1$ \\
\hline $\begin{array}{l}\text { Group C, } \\
\text { lap } 1\end{array}$ & $\mathrm{~A} 1$ & 0 & $\mathrm{Cl}$ & $\mathrm{A} 1+\mathrm{C} 1$ \\
\hline $\begin{array}{l}\text { Group A, } \\
\text { lap 2-5 }\end{array}$ & $\mathrm{A} 1$ & 0 & $\mathrm{C} 2$ & $\mathrm{~A} 1+\mathrm{C} 2$ \\
\hline $\begin{array}{l}\text { Group B, } \\
\text { lap 2-5 }\end{array}$ & $\mathrm{A} 1$ & B1 & $\mathrm{C} 2$ & $\mathrm{~A} 1+\mathrm{B} 1+\mathrm{C} 2$ \\
\hline $\begin{array}{l}\text { Group C, } \\
\text { lap 2-5 }\end{array}$ & $\mathrm{A} 1$ & 0 & $\mathrm{C} 1+\mathrm{C} 2$ & $\mathrm{~A} 1+\mathrm{C} 1+\mathrm{C} 2$ \\
\hline
\end{tabular}

Notice: Al is a variable. Others are constant. All of them are positive.

First, according to the data in Table 1, following results can be reached.

(1) The data of group A in lap 1 have the lowest value.

(2) In laps 2-5, all the four average data (FPRA-R30, FPPB-R30, FPRA-R50, and FPPB-R50) of group A have the lowest value.

(3) The data of group B in laps 2-5 have larger value than data in lap 1.

(4) The data of group C in laps 2-5 have larger value than data in lap 1.

(5) The data of group B have larger value than data of group C in lap 1.

(6) The data of group B have larger value than data of group $\mathrm{C}$ in laps $2-5$.

The results may be explained by the cognition theory and fitness of the model. Seen in Table 2, the confidence values of the information "there is a curve ahead" come from different information channels. Besides, the total value of each group has been defined. A1 is the value of drivers acquiring information from visual channel, which is variable, increased by the vehicle travelling near to the curve. B1 is the value of drivers acquiring warning information from traffic sign. $\mathrm{C} 1$ is the value of drivers acquiring warning information from drivers' memory acquired from the map of scenario.
$\mathrm{C} 2$ is the value of drivers acquiring warning information from drivers' memory acquired by learning system through repeated driving.

According to the basic inequalities, we can safely draw a conclusion as below:

(1) $\mathrm{A} 1<\mathrm{A} 1+\mathrm{B} 1, \mathrm{~A} 1+\mathrm{C} 1, \mathrm{~A} 1+\mathrm{C} 2, \mathrm{~A} 1+\mathrm{B} 1+\mathrm{C} 2, \mathrm{~A} 1+$ $\mathrm{C} 1+\mathrm{C} 2$

(2) $\mathrm{A} 1+\mathrm{C} 2<\mathrm{A} 1+\mathrm{B} 1+\mathrm{C} 2, \mathrm{~A} 1+\mathrm{C} 1+\mathrm{C} 2$

(3) $\mathrm{A} 1+\mathrm{C} 1<\mathrm{A} 1+\mathrm{C} 1+\mathrm{C} 2$

(4) $\mathrm{A} 1+\mathrm{B} 1<\mathrm{A} 1+\mathrm{B} 1+\mathrm{C} 2$

Those inequalities and the cognition assumption can perfectly explain the results of Table 1 . As the assumption argued, the braking operation is the result of confirmation of the information "there is a curve ahead". And to confirm this information the total confidence value must be over limens. Because Al is a variable increasing with driving time. If a positive value is added, the total value can reach the limens earlier, namely, the position of the drivers beginning braking will be away from the curve. Apparently, the inequalities above explained the result (1) to (4), respectively. Indirectly, the result (5) may infer the inequality " $\mathrm{Bl}>\mathrm{Cl}$ ", which can be verified by result (6).

\section{Conclusion}

The information is regarded as the bridge between the cognition model and drivers, because both of sides are working based on the information. Studying the information process and transmit channel can obtain how drivers generate driving operation and build a cognition model to represent driving performance. So the information about curve can be seen as the most crucial factor for driving operation. Based on the cognition model and the results of the simulator experiment, this study will draw the following conclusion.

(1) Both the results of the simulation experiment and the cognition model inference about braking operation show a consistent consequence. Thus, it demonstrates that this cognition model is rational to represent the driving performance at curves. Furthermore, the process of the driver's managing information may be explained by the cognition theory and the characters or working parameters may be acquired by these kinds of simulator experiment. 
TABLE 3: The results of ANOVA analysis.

\begin{tabular}{|c|c|c|c|c|c|c|c|c|c|}
\hline & \multirow{2}{*}{$\begin{array}{c}\text { ANOVA } \\
\text { Lap } 1 \\
\end{array}$} & \multicolumn{2}{|c|}{ FPRA-R30 } & \multicolumn{2}{|c|}{ FPPB-R30 } & \multicolumn{2}{|c|}{ FPRA-R50 } & \multicolumn{2}{|c|}{ FPPB-R50 } \\
\hline & & $F(1,10)$ & $P$ value & $F(1,10)$ & $P$ value & $F(1,10)$ & $P$ value & $F(1,10)$ & $P$ value \\
\hline $1 \#$ & Group A \& B & 28.09 & $0.00^{* *}$ & 10.5 & $0.01^{* *}$ & 6.33 & $0.03^{* *}$ & 6.38 & $0.03^{* *}$ \\
\hline 2 & Group A \& C & 3.57 & $0.09^{*}$ & 0.99 & 0.34 & 6.63 & $0.03^{* *}$ & 6.95 & $0.03^{* *}$ \\
\hline 3 & Group B \& C & 0.01 & 0.91 & 1.66 & 0.23 & 0.17 & 0.69 & 0.31 & 0.59 \\
\hline & Lap 2-5 & $F(1,46)$ & $P$ value & $F(1,46)$ & $P$ value & $F(1,46)$ & $P$ value & $F(1,46)$ & $P$ value \\
\hline $4 \#$ & Group A \& B & 13.94 & $0.00^{* *}$ & 34.51 & $0.00^{* *}$ & 10.356 & $0.00^{* *}$ & 10.58 & $0.00^{* *}$ \\
\hline 5 & Group A \& C & 5.18 & $0.03^{* *}$ & 11.24 & $0.00^{* *}$ & 0.42 & 0.52 & 0.81 & 0.37 \\
\hline 6 & Group B \& C & 1.08 & 0.31 & 3.83 & $0.06^{*}$ & 9.35 & $0.00^{* *}$ & 8.78 & $0.01^{* *}$ \\
\hline & Lap 1, Lap 2-5 & $F(1,28)$ & $P$ value & $F(1,28)$ & $P$ value & $F(1,28)$ & $P$ value & $F(1,28)$ & $P$ value \\
\hline $7 \#$ & $\mathrm{~A}: 1 \& \mathrm{~A}: 2-5$ & 4.86 & $0.04^{* *}$ & 4.86 & $0.04^{* *}$ & 8.23 & $0.01^{* *}$ & 7.06 & $0.01^{* *}$ \\
\hline 8 & $\mathrm{~B}: 1 \& \mathrm{~B}: 2-5$ & 1.00 & 0.33 & 2.62 & 0.12 & 6.51 & $0.02^{*}$ & 2.02 & 0.17 \\
\hline 9 & C:1 \& C:2-5 & 0.07 & 0.79 & 4.13 & $0.05^{* *}$ & 0.41 & 0.53 & 0.92 & 0.35 \\
\hline $10 \#$ & $A: 1 \& B: 2-5$ & 28.03 & $0.00^{* *}$ & 33.45 & $0.00^{* *}$ & 22.23 & $0.00^{* *}$ & 7.06 & $0.01^{* *}$ \\
\hline $11 \#$ & A:1 \& C:2-5 & 11.77 & $0.00^{* *}$ & 14.62 & $0.00^{* *}$ & 18.97 & $0.00^{* *}$ & 22.40 & $0.00^{* *}$ \\
\hline
\end{tabular}

means $P$ value $\leq 0.05 ;{ }^{*} 0.05<P$ value $\leq 0.1$.

TABle 4: The speed of two curves.

\begin{tabular}{lcccccc}
\hline & \multicolumn{2}{c}{ Group A } & \multicolumn{2}{c}{ Group B } & \multicolumn{2}{c}{ Group C } \\
& R30 & R50 & R30 & R50 & R30 & R50 \\
\hline Lap 1 & 11.06 & 13.03 & 9.21 & 11.08 & 10.97 & 11.83 \\
Lap 2-5 & 9.66 & 11.39 & 10.20 & 12.37 & 10.05 & 11.85 \\
\hline
\end{tabular}

(2) The advance warning information can affect the driving decision and make curve driving safer by increasing the confidence value of the curve information. Known the exact process of driving performance, we can modify the driving behavior by changing the information the driver acquired.

(3) There is more than one information channel. Drivers can acquire information via different channels instantaneously. The different information channels may have their own confidence value, and that confidence value may not be constant. Additionally, the information for the perception channel can be stored in knowledge library, or the confidence value of information may be changed by learning system.

\section{Discussion}

Although this pilot study has verified the cognition model in modeling driving performance and explained the information process of drivers, it still has a limitation in clarifying all details of the cognition model and the results of simulation experiment. In this simulation experiment, the impact of driver's ability had been considered and controlled in many ways. However, the personal distinction of different drivers cannot be totally controlled, and the number of the sample size still remains small. Those are the chief causes for deviation and inconsistent result of the ANOVA analysis, seen in Table 3.
We can see that the rows of all the 4 group of data, which have significant differences are $1,4,7,10$, and 11 with a marker of "\#", the $P$ value of which is $\leq 0.05$, and the difference of the confidence between those pairs is large enough. Others do not show a consistent result. The inconsistent may be caused by two kinds of variation coming from drivers. One is that all participants do not have the same ability; however, the analysis assumes that other modules are same during the simplification. The other is that a driver cannot keep the same state in the whole experiment; he/she may take some unpredicted mistakes. Besides, the different curvatures may affect the confidence value of the curve information.

Table 4 shows the entry speed of the curve. Both the two curves have the same result. In lap 1, group A may have the highest entry speed and group B has the lowest entry speed. However, in lap 2-5 the result is contrary. The reason may be the impact from not only the decision module but also the learning system. The analysis of this study only focuses on the braking operation by the decision module at a point time. The entry speed is the result of a sequence of operation about brake and accelerator pedals. Therefore, this involved a series of decisions based on the information. Moreover, it needs further study about other modules of the cognition models and more simulation experiments to acquire the modules working parameters.

\section{Acknowledgment}

This study is supported by the NNSFC project, the study of the mechanism for traffic signs influence on driving behavior and its cognitive model, 51108011.

\section{References}

[1] S. G. Charlton, "The role of attention in horizontal curves: a comparison of advance warning, delineation, and road marking treatments," Accident Analysis \& Prevention, vol. 39, no. 5, pp. 873-885, 2007. 
[2] L. Herrstedt and P. Greibe, "Safer signing and marking of horizontal curves on rural roads," Traffic Engineering and Control, vol. 42, no. 3, pp. 82-87, 2001.

[3] J. E. Hummer, W. Rasdorf, D. J. Findley, C. V. Zegeer, and C. A. Sundstrom, "Curve collisions: road and collision characteristics and countermeasures," Journal of Transportation Safety and Security, vol. 2, no. 3, pp. 203-220, 2010.

[4] T. Ben-Bassat and D. Shinar, "Ergonomic guidelines for traffic sign design increase sign comprehension," Human Factors, vol. 48, no. 1, pp. 182-195, 2006.

[5] W. Wang, Vehicle's Man-Machine Interaction Safety and Driver Assistance, China Communications Press, Beijing, China, 2012.

[6] D. D. Salvucci, "Modeling driver behavior in a cognitive architecture," Human Factors, vol. 48, no. 2, pp. 362-380, 2006.

[7] D. J. Finch, P. Kompfner, C. R. Lockwood, and G. Maycock, "Speed, speed limits and accidents," Project Report 58, Transport Research Laboratory, Crowthorne, UK, 1994.

[8] H. Guo, W. Wang, W. Guo, X. Jiang, and H. Bubb, "Reliability analysis of pedestrian safety crossing in urban traffic environment," Safety Science, vol. 50, no. 4, pp. 968-973, 2012.

[9] S. L. Comte and A. H. Jamson, "Traditional and innovative speed-reducing measures for curves: an investigation of driver behaviour using a driving simulator," Safety Science, vol. 36, no. 3, pp. 137-150, 2000.

[10] I. R. Johnston, "Modifying driver behaviour on rural road curves: a review of recent research," in Proceedings of the 11th Australian Road Research Board (ARRB) Conference, vol. 11, part 4, pp. 115-124, 1982.

[11] D. J. Findley, J. E. Hummer, W. Rasdorf, C. V. Zegeer, and T. J. Fowler, "Modeling the impact of spatial relationships on horizontal curve safety," Accident Analysis \& Prevention, vol. 45, pp. 296-304, 2012.

[12] J. R. Anderson, M. Matessa, and C. Lebiere, "ACT-R: a theory of higher level cognition and its relation to visual attention," Human-Computer Interaction, vol. 12, no. 4, pp. 439-462, 1997.

[13] Y. C. Liu, "A simulated study on the effects of information volume on traffic signs, viewing strategies and sign familiarity upon driver's visual search performance," International Journal of Industrial Ergonomics, vol. 35, no. 12, pp. 1147-1158, 2005.

[14] S. H. Chang, C. Y. Lin, C. P. Fung, J. R. Hwang, and J. L. Doong, "Driving performance assessment: effects of traffic accident location and alarm content," Accident Analysis and Prevention, vol. 40, no. 5, pp. 1637-1643, 2008.

[15] M. Maltz and D. Shinar, "Imperfect in-vehicle collision avoidance warning systems can aid distracted drivers," Transportation Research Part F, vol. 10, no. 4, pp. 345-357, 2007.

[16] G. Abe and J. Richardson, "The influence of alarm timing on braking response and driver trust in low speed driving," Safety Science, vol. 43, no. 9, pp. 639-654, 2005.

[17] J. Roca, C. Castro, M. Bueno, and S. Moreno-Ríos, "A drivingemulation task to study the integration of goals with obligatory and prohibitory traffic signs," Applied Ergonomics, vol. 43, no. 1, pp. 81-88, 2012.

[18] A. Newell, Unified Theories of Cognition, Harvard University Press, Cambridge, Mass, USA, 1990.

[19] W. D. Gray, R. M. Young, and S. S. Kirschenbaum, "Introduction to this special issue on cognitive architectures and humancomputer interaction," Human-Computer Interaction, vol. 12, no. 4, pp. 301-309, 1997.

[20] J. R. Anderson and C. D. Schunn, "Implications of the ACT-R learning theory: no magic bullets," in Advances in Instructional Psychology, R. Glaser, Ed., Erlbaum, Mahwah, NJ, USA, 2000.
[21] A. J. Hornof and D. E. Kieras, "Cognitive modeling demonstrates how people use anticipated location knowledge of menu items," in Proceedings of the ACM Conference on Human Factors in Computing Systems (CHI '99), pp. 410-417, ACM, Pittsburgh, Pa, USA, May 1999.

[22] D. E. Kieras and D. E. Meyer, "The role of cognitive task analysis in the application of predictive models of human performance," in Cognitive Task Analysis, J. M. Schraagen and S. F. Chipman, Eds., pp. 237-260, Erlbaum, Mahwah, NJ, USA, 2000. 


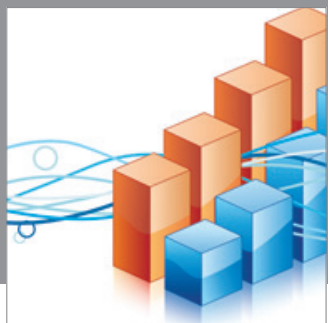

Advances in

Operations Research

mansans



The Scientific World Journal
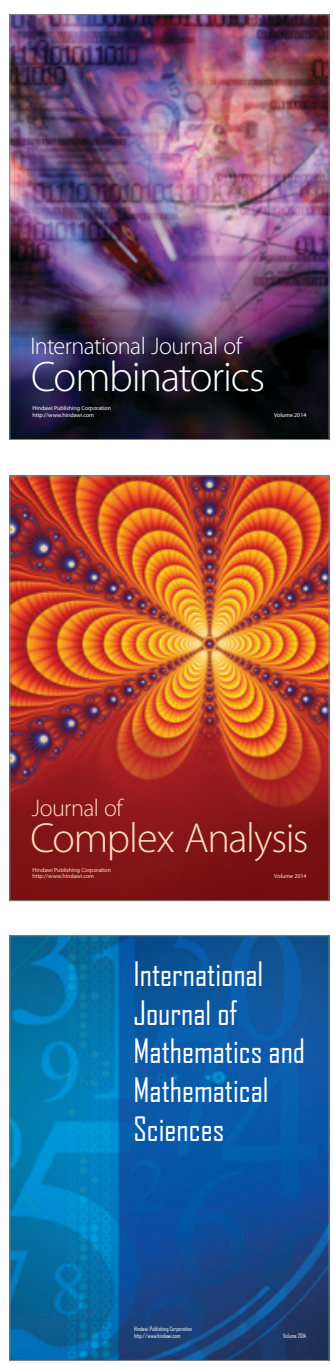
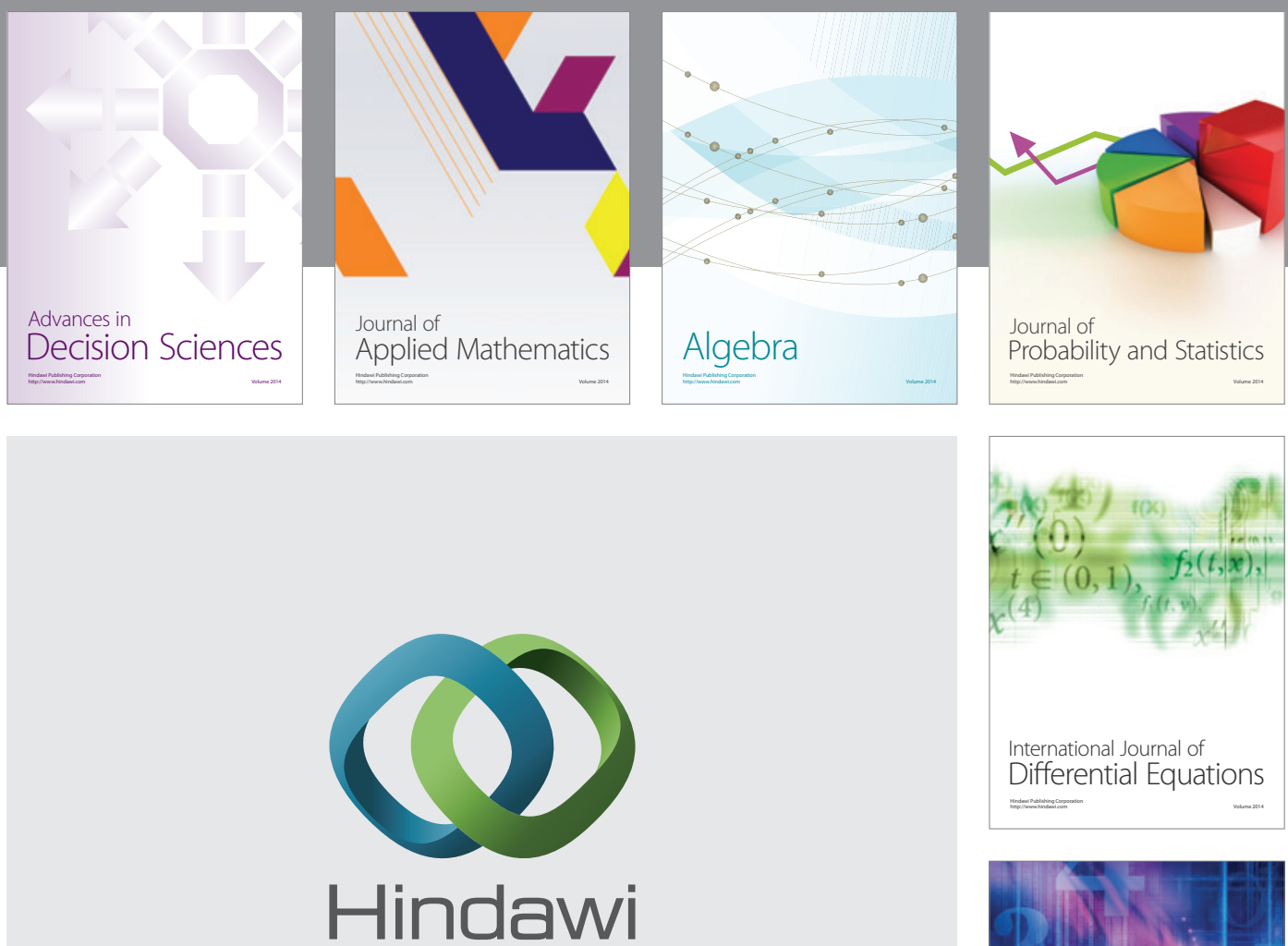

Submit your manuscripts at http://www.hindawi.com
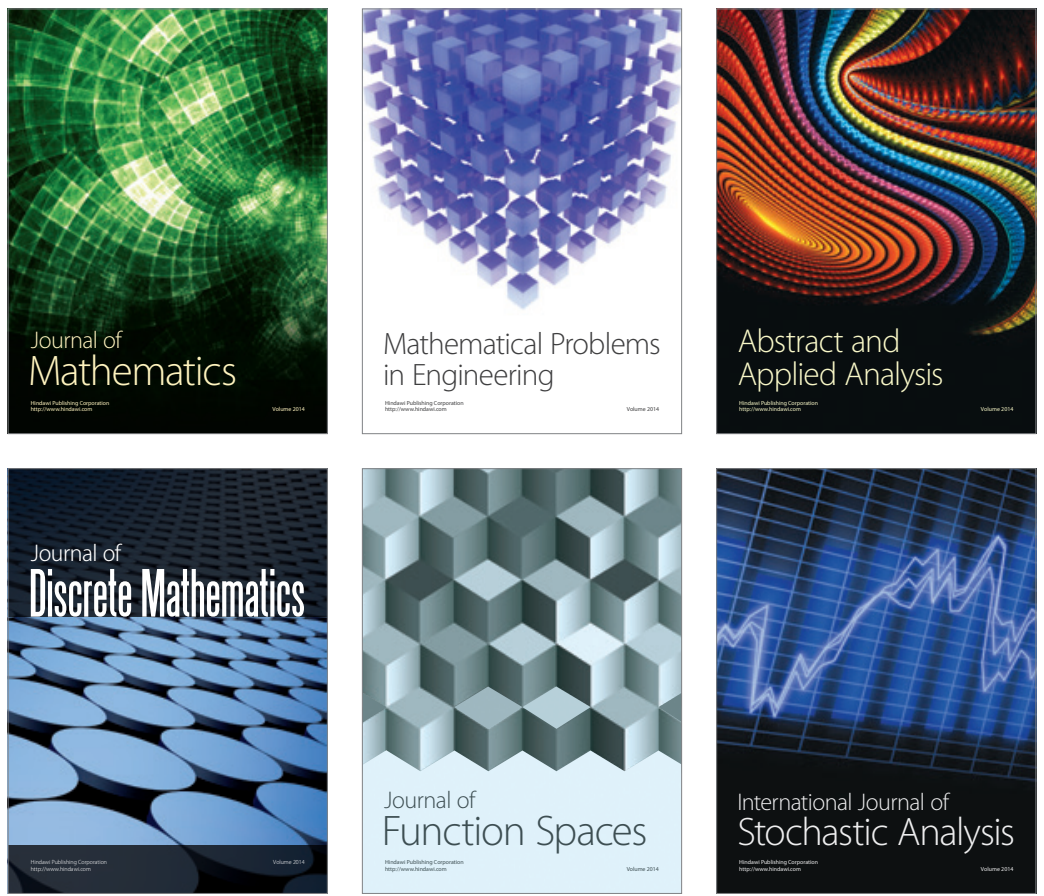

Journal of

Function Spaces

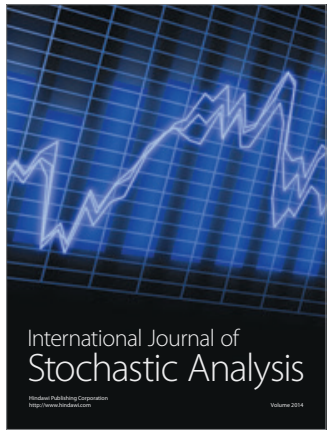

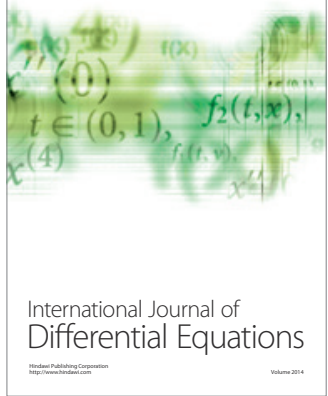
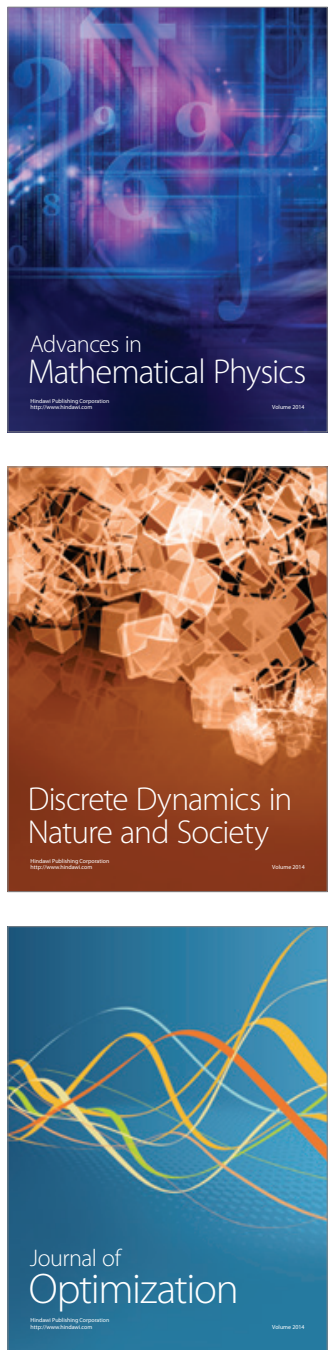